\title{
Pulmonary Hypertension and Thoracic Surgery: Diagnostics and Advances in Therapy and Intraoperative Management
}

\author{
Daisuke F. Nonaka $\cdot$ Katherine P. Grichnik • \\ George B. Whitener
}

Published online: 18 February 2014

(C) Springer Science + Business Media New York 2014

\begin{abstract}
Patients with pulmonary hypertension (PH) remain challenging to care for in the perioperative period. This update will review the most current definitions, the acute and chronic treatment options for $\mathrm{PH}$, including the rationale for various therapeutic agents, the potential side effects and optimization strategies. Anesthetic techniques, which may be affected by PH and/or the medications used to treat $\mathrm{PH}$, are discussed as well as the intraoperative management of $\mathrm{PH}$ with suggestions for addressing adverse events. Specific hemodynamic and metabolic stressors during thoracic surgery, including one-lung ventilation, are emphasized with discussion about the options for intraoperative monitoring. Safe and efficacious care of patients with $\mathrm{PH}$ requires expert cardiovascular management with close attention to medication interactions and hemodynamic perturbations.
\end{abstract}

Keywords Pulmonary hypertension - Pulmonary arterial hypertension · Thoracic surgery

D. F. Nonaka · G. B. Whitener ( $₫)$

Department of Anesthesiology, Duke University Hospital, DUMC Box 3094, 2301 Erwin Road, Durham, NC 27710, USA

e-mail: george.whitener@duke.edu

D. F. Nonaka

e-mail: daisuke.nonaka@duke.edu

\section{K. P. Grichnik}

American Anesthesiology, Mednax National Medical Group,

1301 Concord Terrace, Sunrise, FL 33323, USA

e-mail: katherine_grichnik@americananesthesiology.com

\section{Introduction}

In the past, the traditional definition of pulmonary hypertension $(\mathrm{PH})$ included the following: mean pulmonary artery pressure $(\mathrm{mPAP}) \geq 25 \mathrm{mmHg}$ at rest, $\mathrm{mPAP} \geq 30 \mathrm{mmHg}$ with exercise, pulmonary capillary wedge pressure (PCWP) $\leq 15 \mathrm{mmHg}$ and pulmonary vascular resistance (PVR) $\geq$ 3 Wood units. [The Wood unit can be easily derived from mPAP (measured in $\mathrm{mmHg}$ ) and cardiac output (CO; measured in $1 / \mathrm{min}$ ), and it is thus given in $\mathrm{mmHg} \mathrm{min} / \mathrm{l}$. This is numerically equivalent to hybrid reference units (HRU = Wood units). To convert from Wood units to calculated PVR (dyn $\mathrm{s} / \mathrm{cm}^{5}$ ), the Wood unit must be multiplied by 80 .]

In 2008, however, experts at the Fourth World Symposium on Pulmonary Hypertension simplified the definition. $\mathrm{PH}$ is now defined as $\mathrm{mPAP} \geq 25 \mathrm{mmHg}$ at rest with a normal range for mPAP of $8-20 \mathrm{mmHg}$ [1]. This normal range for mPAP was derived from a systematic literature review of over 1,100 patients from 47 studies and 13 countries, which found normal mPAP to be $14 \pm 3.3 \mathrm{mmHg}$ [2]. The criterion of $\geq 30 \mathrm{mmHg}$ with exercise was removed from the definition, because the same review found almost half of healthy patients $>50$ years old met this parameter.

Also, at the Fourth World Symposium meeting, the clinical classification of $\mathrm{PH}$ was updated to include the following groups [3]:

(1) Pulmonary arterial hypertension (PAH)

(1') Pulmonary veno-occlusive disease and/or pulmonary capillary hemangiomatosis

(2) PH owing to left heart disease

(3) PH owing to lung diseases and/or hypoxia

(4) Chronic thromboembolic pulmonary hypertension

(5) $\mathrm{PH}$ with unclear multifactorial mechanisms 
The gold standard for measuring PAP is right heart catheterization (RHC) [4]. A large-scale study using RHC to categorize these different groups of $\mathrm{PH}$ is not available. However, from a study of 10,314 patients who underwent transthoracic echocardiography (TTE), an estimated systolic PAP $\geq 40 \mathrm{mmHg}$ classified 936 patients as having $\mathrm{PH}$. In this subgroup of PH patients, $68 \%$ were group 2; $9 \%$ were group $3 ; 2.7 \%$ were group $1 ; 2.7 \%$ were group $5 ; 15 \%$ were unclassified [5•].

After the diagnosis of group $1 \mathrm{PH}$, the median survival time is 2.8 years (95\% CI 1.9-3.7 years) [6]. The disease process initially involves chronic vasoconstriction of the pulmonary vessels, followed by smooth muscle hypertrophy and proliferation, and ends with fibrosis at which time the disease becomes fixed [7]. To confirm the diagnosis of $\mathrm{PH}, \mathrm{RHC}$ is indicated. Although RHC is an invasive procedure, a multicenter study of 7,218 catheterizations showed a low mortality rate of $0.055 \%$ or approximately 1 death per 1,800 procedures. The adverse event rate was $1.1 \%$ with the most common complication involving venous access such as hematoma and pneumothorax [8].

\section{Outcome Studies}

There have been many outcome studies on surgical patients with $\mathrm{PH}$, but few have specifically looked at thoracic surgery patients. One study involved $145 \mathrm{PH}$ patients undergoing non-cardiac surgery and found that there was no increased mortality in the thoracic surgery subgroup [9]. However, morbidity was increased in thoracic surgery $(61.5 \%)$ compared to orthopedic procedures $(48 \%)$ and low-risk procedures $(16.7 \%)$, which included gynecologic, urologic, plastic, dermatologic and breast surgery.

Another study of 68 patients with interstitial lung disease undergoing video-assisted thoracic surgery for biopsy reported 3 deaths within 60 days after surgery [10]. Eighteen patients had either an echocardiogram (17 patients) or RHC (1 patient), and 8 had findings consistent with PH. Of these eight patients, four had morbidity. The other ten patients without PH had no morbidity events $(p=0.01)$. In addition, out of the three deaths, two had $\mathrm{PH}$ while the third patient did not have echocardiography or RHC.

A third study reviewed and stratified 96 thoracic surgery patients with $\mathrm{PAH}$ (classification group 1) to mild (systolic PAP $25-44 \mathrm{mmHg}$ ), moderate $(45-59 \mathrm{mmHg})$ and severe $\mathrm{PH}(>60 \mathrm{mmHg}$ ) [11]. Patients with mild and moderate PH had no mortality within 30 days of surgery. However, in patients with severe $\mathrm{PH}, 30$-day mortality was $14 \%$ $(p=0.001)$.

Collectively these studies suggest an increased risk of perioperative morbidity and mortality in $\mathrm{PH}$ patients, especially those with severe $\mathrm{PH}$.
Table 1 Medications and supplements associated with PAH

\begin{tabular}{ll}
\hline Definite & Aminorex \\
& Fenfluramine \\
& Dexfenfluramine \\
& Toxic rapeseed oil \\
& Amphetamines \\
Likely & L-Tryptophan \\
& Methamphetamines \\
Possible & Cocaine \\
& Phenylpropanolamine \\
& St. John's wort \\
& Chemotherapeutic agents \\
& Selective serotonin reuptake inhibitor \\
Unlikely & Oral contraceptives \\
& Estrogen \\
\hline
\end{tabular}

From Simonneau et al. [3]; with permission from Elsevier

\section{Preoperative Assessment}

In addition to RHC, a history and physical examination can assess the risk of perioperative complications. Patient history should include a review of prescribed and illicit medications as well as supplements. Substances associated with PH are listed in Table 1. Clinical signs of right heart failure include dyspnea, increased jugular venous pressure, peripheral edema, ascites and hepatomegaly. An electrocardiogram (ECG) may show signs of right ventricular hypertrophy (tall $\mathrm{R}$ wave in $V_{1}$ with progressive decrease in height from $V_{2}, V_{3}$, to $V_{4}$ ), right axis deviation and right ventricular strain (ST depression in $V_{1}$ ) [12]. Chest X-ray can show enlargement of the proximal pulmonary artery (PA) vasculature with thinning of the peripheral and distal vessels [13]. An arterial blood gas may be used to assess potential hypoxia and metabolic acidosis, which can result from a low CO.

TTE noninvasively evaluates for PH, and several studies have compared its efficacy against RHC. The estimated systolic PAP is commonly used to assess right heart pressures with TTE. In one study, 65 patients who were suspected of PH underwent both TTE and RHC [14]. Using TTE, systolic PAP was calculated by summing the peak pressure of the tricuspid regurgitation jet and right atrial pressure, which was estimated by inferior vena cava diameter and its collapsibility during respiration. They found $48 \%$ of patients had a $>10 \mathrm{mmHg}$ difference between the TTE and RHC measurements. In addition, out of six patients who had no tricuspid regurgitation detected, four had elevated PAP by RHC. The authors concluded that TTE is unreliable for measuring elevated PAP for PH. One can speculate that this may be due to the difficulty in obtaining consistent imaging planes for PAP calculations. 
The above group also analyzed the relationship between survival and another TTE measurement, the tricuspid annular plane systolic excursion (TAPSE) [15]. In 63 patients with $\mathrm{PH}$, they found that estimated survival was significantly decreased in patients with a TAPSE $<1.8 \mathrm{~cm}$ compared to those with a TAPSE $>1.8 \mathrm{~cm}$. At 1 and 2 years, patients with a TAPSE $<1.8 \mathrm{~cm}$ had survival estimates of 57 and $42 \%$, while patients with a TAPSE $>1.8 \mathrm{~cm}$ had survival of 85 and $79 \%$, respectively. The unadjusted risk of death for the TAPSE $<1.8 \mathrm{~cm}$ group was 3.8 (95\% CI 1.6-9.3, $p=0.003$ ).

Using echocardiographic speckle tracking, another group studied patients with PAH (classification group 1) and estimated survival [16]. They found that patients with right ventricular systolic strain less negative than $-12.5 \%$ had more rapid disease progression over 6 months. In addition, these patients had decreased survival over 4 years compared to those with strain more negative than $-12.5 \%$.

With greater interest in diagnosing $\mathrm{PH}$ with noninvasive methods (i.e., decreasing the use of RHC), Bonderman et al. [17] developed a non-invasive algorithm to rule out pre-capillary PH (classification groups 1, 3, 4 and 5). They retrospectively studied 251 patients who were suspected of $\mathrm{PH}$. Even with an estimated systolic PAP of $\geq 36 \mathrm{mmHg}$ by TTE, patients with both the absence of right ventricular strain by ECG and N-terminal brain natriuretic peptide $\leq 80 \mathrm{pg} / \mathrm{ml}$ did not have pre-capillary $\mathrm{PH}$ by RHC. A prospective study of 121 patients validated these findings [17].

\section{Pulmonary Vasodilators}

In patients without $\mathrm{PH}$, innate pulmonary vasodilators (prostacyclin, nitric oxide) and vasoconstrictors (endothelin-1, thromboxane) balance pulmonary vascular tone. $\mathrm{PH}$ patients have an imbalance of these mediators, which leads to pulmonary vasoconstriction, smooth muscle proliferation and local microthrombosis. Current medications to treat $\mathrm{PH}$ manipulate these mediator pathways to decrease PA tone, halt smooth muscle proliferation and decrease thrombosis.

Endogenous nitric oxide (NO), which stimulates guanylate cyclase and increases cyclic guanosine monophosphate (cGMP), triggers vasodilation and decreases platelet aggregation. Synthetic NO is delivered as a continuous inhaled gas and thus selectively impacts pulmonary vasculature without any systemic effects. Because of this selectivity, it reduces shunting by improving $V / Q$ matching and oxygenation. Dosing may range from 5 to $40 \mathrm{ppm}$, although one study showed a ceiling effect at $10 \mathrm{ppm}$ [18]. While inhaled NO is approved by the FDA only for use in neonates with hypoxic respiratory failure associated with PH, many centers extensively utilize nitric oxide as inpatient treatment for adult $\mathrm{PH}$ patients.

Prostacyclin stimulates adenylate cyclase and increases cyclic adenosine monophosphate (cAMP), which promotes vasodilation, increases cardiac contractility and inhibits platelet activation. Prostacyclin medications include IV prostacyclin (half-life $3 \mathrm{~min}$ ), inhaled prostacyclin (half-life $6 \mathrm{~min}$ ), inhaled iloprost (half-life 20-30 min) and subcutaneous treprostinil $[13,19,20]$. Inhaled prostacyclin must be administered continuously, while inhaled iloprost is administered 6-9 times a day because of its longer half-life. IV prostacyclin is reserved for patients with the most severe and difficult to treat $\mathrm{PH}$ [21]. Treprostinil is a continuous subcutaneous infusion, which can be complicated by injection site pain [22]. Compared to NO, inhaled iloprost significantly reduces PAP and PVR and increases CO [23]. Meanwhile, simultaneous delivery of iloprost plus NO and iloprost plus sildenafil exhibit additive effects [24, 25].

Calcium channel blockers (CCB) such as nifedipine, diltiazem and amlodipine are used in a small subset of patients who have PAH and are responders to a trial of NO or IV prostacyclin. A study of patients with $\mathrm{PAH}$ who underwent vasodilator trials with either inhaled NO or IV prostacyclin showed that $12.6 \%$ of patients had vasoreactivity, but only $6.8 \%$ had a long-term clinical response to CCBs [26]. CCB responsiveness is thought to be rare in non-PAH patients (i.e., patients who are not classification group 1) [21].

Phosphodiesterase (PDE) inhibitors include two types, type 3 and 5. Milrinone, enoximone and inamrinone are PDE-3 inhibitors that suppress the breakdown of cAMP, while sildenafil is a PDE-5 inhibitor that suppresses cGMP breakdown. Milrinone increases cardiac contractility, vasodilation and inhibits platelet aggregation. Sildenafil also causes vasodilation and inhibits platelet aggregation, but it increases cardiac contractility via cGMP inhibition of PDE-3 [27]. While milrinone is given IV and causes vasodilation in both the pulmonary and systemic systems, sildenafil's oral vasodilatory effect is mostly limited to the pulmonary vasculature because of low PDE-5 levels in the systemic system except in penile circulation [27].

In patients who require urgent surgery, but have inadequately treated $\mathrm{PH}$, it is recommended to give sildenafil 50-100 mg with L-arginine supplementation [28]. This recommendation is partly due to sildenafil's pulmonary selectivity and uncomplicated route of administration. Theoretically, in a PH patient with left heart disease (classification group 2), sildenafil could cause volume overload of the left heart and lead to pulmonary edema and worsening heart failure. However, a small study of 13 patients with systolic heart failure (7 patients with $\mathrm{PH}, 6$ without $\mathrm{PH}$ ) found that sildenafil decreased PAP without changing $\mathrm{CO}$ or 
PCWP [29]. A separate study investigated sildenafil's effect on 20 patients with chronic obstructive pulmonary disease (COPD; classification group 3) [30]. They found that patients at rest had a significant decrease in $\mathrm{PaO}_{2}$ caused by increased $V / Q$ mismatching and abolition of hypoxic pulmonary vasoconstriction. Furthermore, patients with a higher baseline $\mathrm{PaO}_{2}$ saw a greater decrease in $\mathrm{PaO}_{2}$ after sildenafil compared to those with a lower baseline. Because the drop in $\mathrm{PaO}_{2}$ is significant and unpredictable, sildenafil is not recommended in patients with COPD.

Sildenafil has been associated with blindness in patients being treated for erectile dysfunction by anterior ischemic optic neuropathy (AION) $[31,32]$. Thus, some have recommended withholding electively used sildenafil for 1 week prior to surgery especially if the patient may be exposed to other causes of perioperative blindness such as preoperative anemia, prolonged procedure, substantial blood loss and pressure on the eye $[33,34,35 \bullet$. In contrast, for $\mathrm{PH}$ patients, sildenafil is not an elective medication and must be continued [36]. Therefore, anesthesiologists should be aware of a potential increased risk of perioperative blindness in patients who have recently taken sildenafil (half-life $4 \mathrm{~h}$ ), although there have been no case reports to date of perioperative blindness associated with sildenafil [34].

Endothelin receptor antagonists block two types of receptors, $\mathrm{ET}_{\mathrm{A}}$ and $\mathrm{ET}_{\mathrm{B}}$. $\mathrm{ET}_{\mathrm{A}}$ receptors induce vasoconstriction and smooth muscle proliferation, while $\mathrm{ET}_{\mathrm{B}}$ receptors affect the clearance of endothelin and the secretion of NO and prostacyclin from the endothelium [19]. Bosentan is a nonselective endothelin receptor antagonist, and ambrisentan is $\mathrm{ET}_{\mathrm{A}}$ receptor selective. Both are oral medications with side effects of teratogenicity, elevated transaminase and peripheral edema [19, 21].

In summary, PAH patients (classification group 1) who are positive responders to NO or IV prostacyclin should be placed on a CCB. Non-responders or those who need further treatment after initiating CCB therapy should start an oral medication such as sildenafil or one of the endothelin receptor antagonists. The side effect profile of each drug (sildenafil-decreased platelet function, risk of AION, decreased $\mathrm{PaO}_{2}$ with COPD; ET receptor antagonists-elevated liver transaminase, teratogenicity) should be weighed. For inpatients, one of the inhaled drugs (prostacyclin or iloprost) or IV prostacyclin should be considered depending on illness severity [21]. Figure 1 shows a simplified treatment algorithm for PAH.

\section{Neuraxial Anesthesia}

Many of the vasodilators described above, including NO, prostacyclin-type medications and sildenafil, decrease platelet aggregation. This is concerning, especially as neuraxial anesthesia is commonly used for postoperative pain control after thoracic surgery. There have been no large-scale studies examining the association of these pulmonary vasodilators and risk of epidural hematoma after neuraxial anesthesia; much of the literature is in case report form from obstetrics. Decoene et al. [37] describe a pregnant patient with unexpected $\mathrm{PH}$ who was placed on NO therapy; NO was administered during epidural placement and removal without complications. NO may be a safe choice as its action is selective to the pulmonary vasculature and is rapidly eliminated by hemoglobin (halflife 2-6 s) [19].

Another case report describes a pregnant patient with PH initially treated with IV prostacyclin, which was converted to inhaled prostacyclin [38]. The duration of action of IV prostacyclin on platelet function is $20-30 \mathrm{~min}$. For this patient, an epidural was placed $1 \mathrm{~h}$ after the IV prostacyclin infusion had been stopped. Inhaled prostacyclin continued throughout the intrapartum period, and the epidural was removed after delivery; $2 \mathrm{~h}$ later she was converted back to IV prostacyclin, and there were no complications.

In a third case report, a pregnant patient with PH had an epidural placed [39]. Then intermittent inhaled iloprost was started in the intrapartum period. After delivery, the epidural was removed while the patient continued scheduled intermittent inhaled iloprost. The patient did not develop an epidural hematoma.

There is inadequate evidence to judge the risk of epidural hematoma with these pulmonary vasodilators. Neuraxial anesthesia for patients receiving NO is reasonable given its very short half-life. Both inhaled prostacyclin and iloprost have longer half-lives than NO, but have also been used without neuraxial complications. However, neuraxial anesthesia placement and removal are not recommended for patients receiving IV prostacyclin or IV iloprost $[38,40]$. If a neuraxial technique is strongly indicated, conversion to an inhaled medication should be attempted. If conversion is successful, then the anesthesiologist may consider proceeding with neuraxial anesthesia after weighing the risks and benefits. One should wait at least $2 \mathrm{~h}$ after the discontinuation of the prostacyclin or iloprost infusion before neuraxial placement to allow the recovery of platelet function [40]. There are no published case reports of neuraxial anesthesia with sildenafil.

\section{Ventricular Support: Vasopressors, Inotropes and Intra-Aortic Balloon Pump (IABP)}

Astute cardiovascular management of $\mathrm{PH}$ calls for adequate myocardial perfusion and limits oxygen consumption of the right heart by minimizing right ventricular end- 
Fig. 1 Simplified treatment algorithm for $\mathrm{PAH}$

(classification group 1). $\mathrm{NO}$ nitric oxide, $I V$ intravenous, $C C B$ calcium channel blocker, $E R A$ endothelin receptor antagonist, $P H$ pulmonary hypertension, $A I O N$ anterior ischemic optic neuropathy, $C O P D$ chronic obstructive pulmonary disease

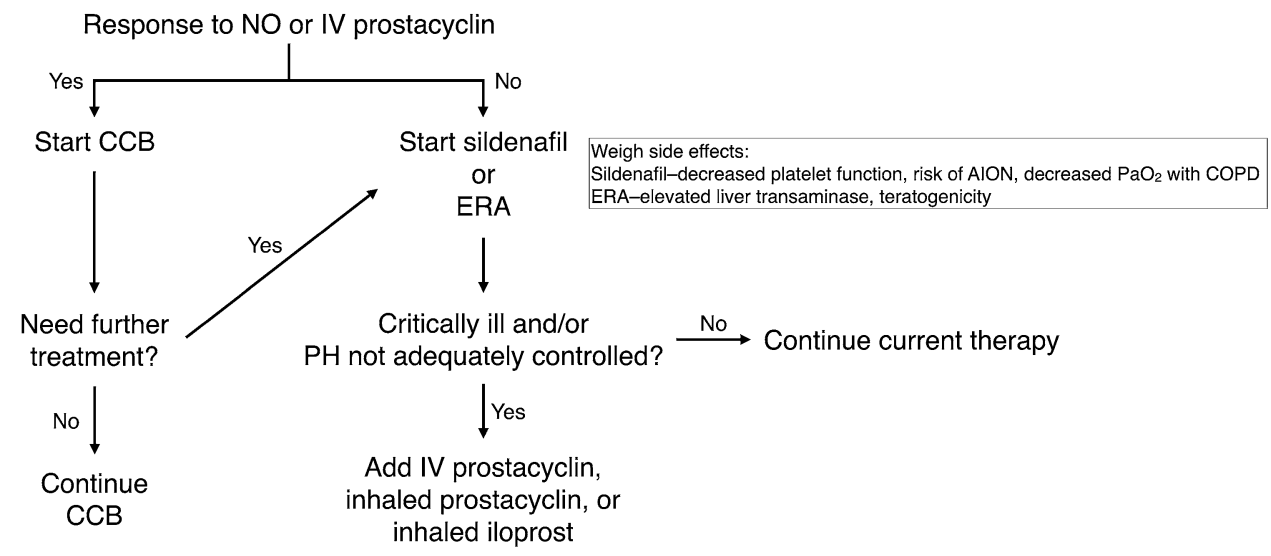

diastolic volume and wall stress. Right myocardial perfusion is the difference between systemic arterial pressure and right ventricular pressure; perfusion can be optimized by increasing systemic pressure using vasopressors such as phenylephrine, norepinephrine and vasopressin, while decreasing right ventricular end-diastolic pressure and volume with inotropes such as milrinone, epinephrine and dobutamine. Inotropes also increase myocardial perfusion by increasing $\mathrm{CO}$.

Norepinephrine is a more suitable drug than phenylephrine in PH patients, as it not only increases systemic pressure via alpha receptors, but also increases contractility and $\mathrm{CO}$ via beta receptors. Norepinephrine increases $\mathrm{CO}$ during acute increases of PAP such as initiation of onelung ventilation $(\mathrm{OLV})$ in thoracic surgery, but has minimal CO effect in patients with chronic PH [41]. Vasopressin is also recommended in patients with $\mathrm{PH}$. Animal models have demonstrated that vasopressin causes systemic vasoconstriction and pulmonary vasodilation by triggering NO release from the pulmonary endothelium [42].

Of the inotropes, milrinone is commonly used in patients with $\mathrm{PH}$ for its contractility and pulmonary vasodilatation effects via increasing available cAMP. However, caution must be exercised with milrinone, as it also decreases systemic pressure and can lead to decreased myocardial perfusion. Epinephrine and dobutamine are inotropes that can also be used as long as tachycardia is avoided. Tachycardia leads to increased oxygen consumption and decreased diastolic myocardial perfusion time in patients with right ventricular hypertrophy.

IABP augments myocardial perfusion during diastole and supplements pharmacologic support. In a study of ten pigs, right ventricular failure was induced by artificial PA constriction [43]. Dosing of phenylephrine and hemodynamic parameters were measured with and without IABP. Results showed that with IABP, the pigs required less phenylephrine to maintain systemic pressures and had increased augmentation of myocardial perfusion pressures.
In addition, IABP resulted in an increase in cardiac index and mixed venous oxygen saturation due to decreased systemic afterload.

\section{Intraoperative Management}

The intraoperative management of $\mathrm{PH}$ patients undergoing thoracic surgery involves maintaining right heart myocardial perfusion and decreasing oxygen consumption as stated above. Key times when the right heart is at risk include induction, initiation of positive pressure ventilation, initiation of OLV, and clamping of the PA during pneumonectomy and lung transplantation. During induction, systemic pressures may need support with vasopressors to preserve myocardial perfusion. Positive pressure ventilation compresses intra-alveolar vasculature and increases PAP and PVR. OLV increases PAP, PVR and right ventricular workload by causing hypoxic pulmonary vasoconstriction. Finally, clamping PA branches increases PAP, PVR and myocardial oxygen consumption by acutely decreasing the pulmonary vascular bed area. In addition to vigilance during these key times, the overall anesthetic plan should avoid other triggers that would increase PVR. These triggers include hypoxemia, hypercarbia, acidosis, hypervolemia, hypothermia and sympathetic stimulation (pain).

Ventilation and tidal volumes should be adjusted to keep lung volumes near functional residual capacity as this is the point where PVR is lowest. Attempts to avoid hyperinflation and atelectasis should be made as both lead to compression of alveolar vessels causing increased PVR [13]. When OLV is initiated, tidal volumes should be decreased by half because functional residual capacity also decreases by half. It is recommended that in the postoperative period, mechanically ventilated $\mathrm{PH}$ patients should have ventilator settings similar to those of acute respiratory distress syndrome (tidal volume $6 \mathrm{ml} / \mathrm{kg}$ and plateau pressure $\leq 30 \mathrm{~cm} \mathrm{H}_{2} \mathrm{O}$ ) [44].

Vasodilators should also be used as necessary for intraoperative management in thoracic surgery. As 
mentioned previously, in urgent cases of patients with inadequate workup for $\mathrm{PH}$, sildenafil 50-100 $\mathrm{mg}$ should be considered even for classification group 2 patients, but not group $3[28,30]$. Other pulmonary vasodilators including NO, milrinone and the prostacyclin-type medications should be used with caution in patients who are classification group $1^{\prime}$ and 2 , as it may lead to pulmonary edema and/or left heart failure [45].

A pulmonary artery catheter (PAC) is still widely used for patients with $\mathrm{PH}$ in the perioperative period. Physicians view it as a reliable way to follow cardiac function by measuring central venous pressure (CVP), PAP, CO and mixed venous oxygen saturation. Further, the ability to trend both CVP and CO simultaneously may more efficiently demonstrate changes in right ventricular function than PAP [41]. However, a multicenter randomized trial of approximately 2,000 elderly patients showed that there was no difference in benefit or mortality between patients who were treated with and without a PAC [46].

Transesophageal echocardiography (TEE) is increasingly being used as a perioperative monitor in patients to upplement or even replace a PAC. TEE can assess for tricuspid regurgitation, right ventricular function and interventricular septal bounce [47•]. Interventricular septal function can distinguish between pressure or volume overload of the right heart. If the interventricular septum bows to the left during systole, pressure overload is likely; if the septum bows to the left during diastole, volume overload is suspected. Bowing of the septum to the left is especially detrimental during diastole because this encroachment decreases left ventricular end diastolic volume and impairs $\mathrm{CO}$. With a diminished $\mathrm{CO}$ and an overloaded right ventricle, right heart myocardial perfusion is greatly compromised. In major thoracic procedures such as pneumonectomy and lung transplantation, TEE is considered superior to the PAC [47•]. For lower risk thoracic surgeries (wedge resections and lobectomies done by either thoracoscopy or thoracotomy), TEE is rarely indicated.

There are individual benefits to PAC and TEE. PAC monitoring provides straightforward numerical data in distinction to TEE, for which a video loop must be obtained and interpreted by a specialist. Therefore, a PAC may be more suitable for continuous monitoring, because it is much simpler to trend numerical values than TEE loops over time. Also, a PAC allows quick calculation of right heart myocardial perfusion pressure by subtraction of systemic from pulmonary pressure. On the other hand, TEE has the benefit of simultaneous right and left heart evaluation [47•]. In addition, TEE allows for better assessment of intraoperative hypotension by evaluating for wall motion abnormalities, volume status, tamponade and large pulmonary thrombus.

\section{Conclusion}

Anesthetic management of thoracic surgery patients with PH initially involves identifying the cause and classification of $\mathrm{PH}$ as certain medical therapies are contraindicated in different groups. After careful patient workup, including a review of medications, physical status and preoperative tests, the anesthesiologist should plan for key intraoperative events such as initiation of OLV and PA clamping. Invasive monitoring such as PAC and TEE should be considered, and vasoactive drugs should be readily available to treat sudden hemodynamic changes. Extensive knowledge of cardiac physiology and pharmacology of $\mathrm{PH}$ medications is needed to take care of these patients during their perioperative course.

\section{Compliance with Ethics Guidelines}

Conflict of Interest Daisuke F. Nonaka, Katherine P. Grichnik and George B. Whitener declare that they have no conflict of interest.

Human and Animal Rights and Informed Consent This article does not contain any studies with human or animal subjects performed by any of the authors.

\section{References}

Papers of particular interest, published recently, have been highlighted as:

- Of importance

1. Badesch DB, Champion HC, Sanchez MA, et al. Diagnosis and assessment of pulmonary arterial hypertension. J Am Coll Cardiol. 2009;54:S55-66.

2. Kovacs G, Berghold A, Scheidl S, et al. Pulmonary arterial pressure during rest and exercise in healthy subjects: a systematic review. Eur Respir J. 2009;34:888-94.

3. Simonneau G, Robbins IM, Beghetti M, et al. Updated clinical classification of pulmonary hypertension. J Am Coll Cardiol. 2009;54:S43-54.

4. Galie N, Hoeper MM, Humbert M, et al. Guidelines for the diagnosis and treatment of pulmonary hypertension: the Task Force for the Diagnosis and Treatment of Pulmonary Hypertension of the European Society of Cardiology (ESC) and the European Respiratory Society (ERS), endorsed by the International Society of Heart and Lung Transplantation (ISHLT). Eur Heart J. 2009;30:2493-537.

5. - Strange G, Playford D, Stewart S et al. Pulmonary hypertension: prevalence and mortality in the Armadale echocardiography cohort. Heart. 2012;98:1805-11. Extensive study $(>10,000$ patients) characterizing the prevalence of pulmonary hypertension and its classification subgroups.

6. D'Alonzo GE, Barst RJ, Ayres SM, et al. Survival in patients with primary pulmonary hypertension. Results from a national prospective registry. Ann Intern Med. 1991;115:343-9.

7. Fox C, Kalarickal PL, Yarborough MJ, et al. Perioperative management including new pharmacological vistas for patients 
with pulmonary hypertension for noncardiac surgery. Curr Opin Anaesthesiol. 2008;21:467-72.

8. Hoeper MM, Lee SH, Voswinckel R, et al. Complications of right heart catheterization procedures in patients with pulmonary hypertension in experienced centers. J Am Coll Cardiol. 2006;48: 2546-52.

9. Ramakrishna G, Sprung J, Ravi BS, et al. Impact of pulmonary hypertension on the outcomes of noncardiac surgery: predictors of perioperative morbidity and mortality. J Am Coll Cardiol. 2005;45:1691-9.

10. Kreider ME, Hansen-Flaschen J, Ahmad NN, et al. Complications of video-assisted thoracoscopic lung biopsy in patients with interstitial lung disease. Ann Thorac Surg. 2007;83:1140-4.

11. Jurado JE, Bacchetta MD, Ginsburg ME, et al. Safety of thoracic surgery in patients with pulmonary hypertension. In: 92nd annual meeting of the American Association for Thoracic Surgery. San Francisco: Moscone West Convention Center; 2012.

12. Nauser TD, Stites SW. Diagnosis and treatment of pulmonary hypertension. Am Fam Phys. 2001;63:1789-98.

13. Subramaniam K, Yared JP. Management of pulmonary hypertension in the operating room. Semin Cardiothorac Vasc Anesth. 2007;11:119-36.

14. Fisher MR, Forfia PR, Chamera E, et al. Accuracy of Doppler echocardiography in the hemodynamic assessment of pulmonary hypertension. Am J Respir Crit Care Med. 2009;179:615-21.

15. Forfia PR, Fisher MR, Mathai SC, et al. Tricuspid annular displacement predicts survival in pulmonary hypertension. Am J Respir Crit Care Med. 2006;174:1034-41.

16. Sachdev A, Villarraga HR, Frantz RP, et al. Right ventricular strain for prediction of survival in patients with pulmonary arterial hypertension. Chest. 2011;139:1299-309.

17. Bonderman D, Wexberg P, Martischnig AM, et al. A noninvasive algorithm to exclude pre-capillary pulmonary hypertension. Eur Respir J. 2011;37:1096-103.

18. Solina AR, Ginsberg SH, Papp D, et al. Dose response to nitric oxide in adult cardiac surgery patients. J Clin Anesth. 2001;13: 281-6.

19. MacKnight B, Martinez EA, Simon BA. Anesthetic management of patients with pulmonary hypertension. Semin Cardiothorac Vasc Anesth. 2008;12:91-6.

20. Siobal M. Aerosolized prostacyclins. Respir Care. 2004;49:640-52.

21. Badesch DB, Abman SH, Simonneau G, et al. Medical therapy for pulmonary arterial hypertension: updated ACCP evidencebased clinical practice guidelines. Chest. 2007;131:1917-28.

22. Gille J, Seyfarth HJ, Gerlach S, et al. Perioperative anesthesiological management of patients with pulmonary hypertension. Anesthesiol Res Pract. 2012;2012:356982.

23. Winterhalter M, Simon A, Fischer S, et al. Comparison of inhaled iloprost and nitric oxide in patients with pulmonary hypertension during weaning from cardiopulmonary bypass in cardiac surgery: a prospective randomized trial. J Cardiothorac Vasc Anesth. 2008; 22:406-13.

24. Flondor M, Merkel M, Hofstetter C, et al. The effect of inhaled nitric oxide and inhaled iloprost on hypoxaemia in a patient with pulmonary hypertension after pulmonary thrombarterectomy. Anaesthesia. 2006;61:1200-3.

25. Wilkens H, Guth A, Konig J, et al. Effect of inhaled iloprost plus oral sildenafil in patients with primary pulmonary hypertension. Circulation. 2001;104:1218-22.

26. Sitbon $\mathrm{O}$, Humbert $M$, Jais $X$, et al. Long-term response to calcium channel blockers in idiopathic pulmonary arterial hypertension. Circulation. 2005;111:3105-11.

27. Archer SL, Michelakis ED. Phosphodiesterase type 5 inhibitors for pulmonary arterial hypertension. N Engl J Med. 2009;361:1864-71.
28. Blaise G, Langleben D, Hubert B. Pulmonary arterial hypertension: pathophysiology and anesthetic approach. Anesthesiology. 2003;99:1415-32.

29. Lewis GD, Lachmann J, Camuso J, et al. Sildenafil improves exercise hemodynamics and oxygen uptake in patients with systolic heart failure. Circulation. 2007;115:59-66.

30. Blanco I, Gimeno E, Munoz PA, et al. Hemodynamic and gas exchange effects of sildenafil in patients with chronic obstructive pulmonary disease and pulmonary hypertension. Am J Respir Crit Care Med. 2010;181:270-8.

31. Pomeranz HD, Smith KH, Hart WM Jr, et al. Sildenafil-associated nonarteritic anterior ischemic optic neuropathy. Ophthalmology. 2002;109:584-7.

32. Cunningham AV, Smith KH. Anterior ischemic optic neuropathy associated with viagra. J Neuroophthalmol. 2001;21:22-5.

33. Fodale V, Di Pietro R, Santamaria S. Viagra, surgery and anesthesia: a dangerous cocktail with a risk of blindness. Med Hypotheses. 2007;68:880-2.

34. McAllister RK, Meyer TA, Bittenbinder TM. Are guidelines needed for the perioperative discontinuation of phosphodiesterase type 5 inhibitors? J Clin Anesth. 2008;20:560-1.

35. Practice advisory for perioperative visual loss associated with spine surgery: an updated report by the American Society of Anesthesiologists Task Force on perioperative visual loss. Anesthesiology. 2012;116:274-85. This 2012 practice advisory updates the 2006 version. However, after reviewing the most recent literature the committee did not find it necessary to modify the recommendations from the 2006 version.

36. Edwards AF, Roy RC. Preoperative administration of PDE-5 Inhibitors. J Clin Anesth. 2009;21:149; author reply 149-50.

37. Decoene C, Bourzoufi K, Moreau D, et al. Use of inhaled nitric oxide for emergency Cesarean section in a woman with unexpected primary pulmonary hypertension. Can J Anaesth. 2001;48:584-7.

38. Bildirici I, Shumway JB. Intravenous and inhaled epoprostenol for primary pulmonary hypertension during pregnancy and delivery. Obstet Gynecol. 2004;103:1102-5.

39. Weiss BM, Maggiorini M, Jenni R, et al. Pregnant patient with primary pulmonary hypertension: inhaled pulmonary vasodilators and epidural anesthesia for cesarean delivery. Anesthesiology. 2000;92:1191-4.

40. Pittman J, Dunnet JM. Central nerve block and thromboprophylaxis. Br J Anaesth. 1999;83:689-90.

41. Forrest P. Anaesthesia and right ventricular failure. Anaesth Intensive Care. 2009;37:370-85.

42. Evora PR, Pearson PJ, Schaff HV. Arginine vasopressin induces endothelium-dependent vasodilatation of the pulmonary artery. V1-receptor-mediated production of nitric oxide. Chest. 1993; 103:1241-5.

43. Liakopoulos OJ, Ho JK, Yezbick AB, et al. Right ventricular failure resulting from pressure overload: role of intra-aortic balloon counterpulsation and vasopressor therapy. J Surg Res. 2010;164:58-66.

44. Hill NS, Roberts KR, Preston IR. Postoperative pulmonary hypertension: etiology and treatment of a dangerous complication. Respir Care. 2009;54:958-68.

45. Ross AF, Ueda K. Pulmonary hypertension in thoracic surgical patients. Curr Opin Anaesthesiol. 2010;23:25-33.

46. Sandham JD, Hull RD, Brant RF, et al. A randomized, controlled trial of the use of pulmonary-artery catheters in high-risk surgical patients. N Engl J Med. 2003;348:5-14.

47. - Sullivan B, Puskas F, Fernandez-Bustamante A. Transesophageal echocardiography in noncardiac thoracic surgery. Anesthesiol Clin. 2012;30:657-69. A review of current monitoring practices in thoracic surgery and the use of TEE for pneumonectomy, aortic surgery, lung transplantation and initiation of ECMO. 